\title{
HEPATITIS B VACCINATION STATUS IN HEALTH CARE WORKERS OF TWO UNIVERSITY HOSPITALS
}

\author{
Muhammad S Memon, Shoaib Ansari, Rashid Nizamani, Naresh Kumar Khatri, \\ Mukhtar A Mirza and Wasim Jafri
}

\begin{abstract}
BACKGROUNDI OBJECTIVE: Hepatitis B virus (HBV) infection is the leading cause of hepatocellular carcinoma and acute liver failure in Asia. It usually transmits through parenteral routes. The health personnel are at high risk for acquiring HBV infection. This study was designed to determine the immunization status for HBV infection in healthcare workers (HCWs) of two university hospitals in Sindh, Pakistan.

METHODS: The healthcare workers of two university hospitals (Liaquat University Hospital, Jamshoro and Isra University Hospital, Hyderabad) were selected and divided into ten different groups.

RESULTS: There were total 923 participants including 649 (70\%) males and $274(30 \%)$ females. The mean age of participants was 29.6 years. A total of $596(64.6 \%)$ participants were immunized for HBV infection and $392(66.2 \%)$ were inoculated three or more than three doses of vaccine. The pre vaccination HBSAg status was checked in $380(41.2 \%)$ and it was positive in 18 (4.7\%) participants. The frequency of immunization was highest in doctors (92.4\%) and lowest in nursing assistants (18.9\%).

CONCLUSION: A good number of HCWs is vaccinated against HBV infection in these two university hospitals of Sindh compared to general population of Pakistan. This study shows the need for a more aggressive approach to get HCWs vaccinated, because a significant percentage of them is not protected.
\end{abstract}

KEY WORDS: Hepatitis B. Immunization. Healthcare Workers.

\section{INTRODUCTION}

Approximately $30 \%$ of world population has serological evidence of hepatitis B virus (HBV) infection. Of these, an estimated 350 million have chronic HBV infection and at least 500,000 chronically infected persons die each year from liver cancer and cirrhosis. ${ }^{1}$ $\mathrm{HBV}$ is the greatest threat of infection for health care workers (HCWs). The risk of contracting HBV by $\mathrm{HCWs}$ is four times greater than that of general adult population, among those who do not work / are not a part of health care institutions. ${ }^{2}$ A safe and effective vaccine against HBV is available for nearly 20 years. Hepatitis B vaccine is effective in preventing HBV infections when it is given either before or shortly after exposure. At least $85-90 \%$ of HBV associated deaths are vaccine preventable. ${ }^{1}$ Risk of acquiring HBV infection and status of Hepatitis B vaccine in developing countries have been poorly quantified, particularly the data regarding the risk to different categories of HCWs, like nurses and supporting nonprofessional staffs, which clean the wards and instruments, is scarce. ${ }^{3}$ The present study was conducted to assess the vaccination status of HCWs and to iden- tify the reasons for not getting vaccinated.

\section{METHODS}

Our descriptive study used a self administered precoded and pre-tested questionnaire, consisting of designation of respondent and questions regarding status of hepatitis B vaccination. The questionnaire was given to a cross section of HCWs in two university hospitals (Liaquat University Hospital, Jamshoro and Isra University Hospital, Hyderabad) in Sindh, Pakistan. A verbal consent was obtained from each of the participant. The participants were doctors, staff nurses, medical students, nursing students, nursing assistants, operation theater (OT) technicians, laboratory technicians, X-ray technicians, hospital administration staff and house keeping staff. The study was conducted in January 2005. Data was entered and analyzed in SPSS 11.0 version. The $X^{2}$ (chi-square) test and independent t-test, as required by the sample size were used to assess the significance of difference. All the available information on each variable was used. P value of 0.05 or less was considered to indicate statistical significance. 


\section{RESULTS}

A total of 1610 participants was working in both university hospitals received the questionnaire and 923 completed. The frequency of various working groups is depicted in Table I. The mean age of the participants was 29.6 years. Six hundred and forty nine (70\%) were men and two seventy four (30\%) were women. A total of $18(4.72 \%)$ HCWs were found to have HBSAg positive and all were not vaccinated. A total of 589 persons (64\%) were vaccinated against HBV infection. Out of those only 392 (66.5\%) HCWs had completed their vaccination (three or more than three doses). There was no difference in vaccination status of men and women.

There was a significant difference in mean ages of vaccinated and not vaccinated HCWs. Highest frequency of vaccination was seen in doctors (92.4\%) and least in nursing assistants (17.2\%). A statistically significant difference $(P=0.04)$ was noted in vaccination status of various working groups. Those HCWs who were more experienced had better vaccination status ( $p$ value 0.08). There was no difference in total number of pricks during the job tenure in vaccinated and not vaccinated groups. A significant difference $(P<0.00)$ was noted in practice of checking prevaccination HBSAg status in vaccinated and nonvaccinated group.

The reasons given by $\mathrm{HCW}$ who did not get themselves vaccinated were lack of interest $46.7 \%$ $(n=156)$, lack of knowledge regarding the importance of HBV prevention 20\% ( $n=67)$, high cost $15.6 \%$ $(n=52)$, did not mention any reason $8.4 \%(n=28)$, HBSAg positive status 5.2\%(n=18), fear about positive test for HBSAg $2.2 \%(n=7)$ and history of previous jaundice $1.8(n=6)$.

TABLE I:

CHARACTERISTICS OF THE 923 PARTICIPANTS STRATIFIED BY HBV VACCINATION STATUS

\begin{tabular}{|l|c|c|c|}
\hline & Vaccinated (n = 589) & Not vaccinated (n = 334) & p- value \\
\hline Age & $30.38 \pm 9.01$ & $28.24 \pm 9.8$ & 0.001 \\
\hline Sex & & & 0.74 \\
\hline Men & $70 \%$ & $30 \%$ & \\
\hline Women & $30 \%$ & $70 \%$ & 0.04 \\
\hline Working Groups (n) & & & \\
\hline OT Technicians (84) & $54.8 \%$ & $45.2 \%$ & \\
\hline Nurses (56) & $69.6 \%$ & $30.4 \%$ & \\
\hline Doctors (158) & $92.4 \%$ & $7.6 \%$ & \\
\hline Nursing Assistants (58) & $17.2 \%$ & $82.8 \%$ & \\
\hline Lab. Technicians (63) & $66.7 \%$ & $33.3 \%$ & \\
\hline X-ray Technicians (43) & $48.8 \%$ & $51.2 \%$ & \\
\hline Hosp. Management (87) & $47.1 \%$ & $52.9 \%$ & \\
\hline Medical Students (180) & $79.4 \%$ & $20.6 \%$ & \\
\hline Nursing Students (91) & $39.6 \%$ & $60.4 \%$ & \\
\hline Housekeeping staff (103) & $63.1 \%$ & $36.9 \%$ & \\
\hline & & & \\
\hline Duration of Work (years) & $7.9 \pm 8.6$ & $6.9 \pm 8.5$ & \\
\hline Total number of pricks & $4.92 \pm 9.6$ & 0.00 & \\
\hline Pre-vaccine HBSAg checked & & $24.5 \%$ & \\
\hline Yes & $50.7 \%$ & $79.3 \%$ & \\
\hline No & & & \\
\hline
\end{tabular}




\section{DISCUSSION}

Health Care Workers have a greater probability of acquiring Hepatitis B infection, because they are occupationally exposed. The need for vaccination against this disease should be considered a priority. HBV vaccine is highly effective with 95\% seroconversion rates. 4

Our study revealed that $64 \%(n=589)$ of the HCWs were vaccinated, while Nilofer et al, Nasir et al and Younus et al have reported vaccination rate of $86 \%$, $49 \%$ and $72 \%$ respectively among the $\mathrm{HCWs}$. ${ }^{5,6,7}$ Compared to above quoted studies, we have better vaccination rate than reported by Nasir et al. The vaccination rate in our study is quite comparable to studies reported from Brazil, ${ }^{8}$ Iran $^{9}$ and Nepal ${ }^{3}$ which is $79 \%, 62 \%$ and $48.9 \%$ respectively. There was no difference in vaccination status of men and women in our and above reported studies.

There was significant difference in rate of vaccination in various working groups. The highest vaccination rate was seen in doctors (92.4\%) and least in nursing assistants (17.2\%). Similar results of vaccination rates were reported by Nilofer et al, ${ }^{5} \mathrm{SF}$ Imam et al, ${ }^{10} \mathrm{SK}$ Surestha et $\mathrm{al}^{3}$ and Nasir $\mathrm{K}$ et al. ${ }^{6}$ They all reported high rate in doctors than other paramedical staff. Surprisingly, the housekeeping staff had better vaccination status than nursing students, X-ray and O.T. Technicians, hospital management staff, and nursing assistants as shown in Table I. We could not find the reason for this disparity, even the housekeeping staff was least educated among the all categories we made. This phenomenon is also reported by Nilofer et al. ${ }^{5}$ She reported better vaccination rate of housekeeping staff (90\%) than laboratory and O.T. Technicians (78\%) at the Aga Khan University Hospital, Karachi.

We also found that the advance age (mean 30 years) and increased working experience (mean 8 years) were significant factors in higher rate of vaccination. These factors suggest that more experience in working as a hospital staff reflects more awareness regarding various communicable diseases.

The prevalence of HBSAg positive status was $4.72 \%$ $(18 / 923)$ in our study which is quite higher than healthy blood donors $(1351 / 64720=2 \%)$ of Liaquat University Hospital from 2001 to $2004 .{ }^{11}$ The prevalence of HBSAg positive status in various HCWs studies from Pakistan is 5 to $9 \% .^{12,16}$ We have same prevalence as reported in other parts of Pakistan.

Though, pre-vaccination checking of HBSAg status is not mandatory for mass vaccination, here we noted that those who checked it prior to vaccination had better compliance with it compared to those who did not check it. This shows that those HCWs are keen in knowing their HBSAg status prior to vaccination and on getting negative result they get vaccinated.

Despite the availability of vaccination for more than two decades, $100 \%$ coverage of vaccination has not yet been achieved. The most frequently quoted reason amongst the non -vaccinated HCWs in this study was the ignorance ( lack of interest and lack of knowledge) about the importance of HBV prevention. Same reason was reported in the study done at The Aga Khan University Hospital, Karachi. ${ }^{5}$ Whereas, studies from Fatima Jinnah Medical College ${ }^{6}$ and Allama Iqbal Medical College ${ }^{7}$ Lahore have cited the main reason for not to vaccinate the high cost of vaccine.

\section{CONCLUSION}

A good number of HCWs is vaccinated against HBV infection in these two university hospitals of Sindh compared to general population of Pakistan. Our study shows the need for a more aggressive approach to get HCWs vaccinated, because a significant percentage of them is not protected.

\section{REFERENCES}

1. Document from Department of vaccines and biological, World Health Organization 1211 Geneva, 2001. Available at: www.who.int/vaccinesdocumetns/.

2. Byrne EB. Viral hepatitis: an occupational hazard of medical personnel. Experience of the Yale new Haven Hospital, 1952 to 1965. JAMA. 1966; 195 (5): 362-4.

3. Surestha SK, Bhattarai MD. Study of hepatitis B among different categories of health care workers. JCPSP. 2006; 16(2): 108-111.

4. Update on hepatitis B prevention. MMWR Morb Mortal Wkly Rep. 1987; 36:353-60.

5. Ali NS, Jamal K and Qureshi R. Hepatitis B vaccination status and identification of risk factors for hepatitis B in health care workers. JCPSP. 2005; 15(5): 257-260.

6. Nasir K, Khan KA, Kadri WM, Salim S, Tufail K, Sheikh HA, et al. Hepatitis $B$ vaccination among health care workers and students of a medical college. J Pak Med Assoc. 2000; 50:239-43.

7. Younus BB, Khan GM, Akhtar P, Chudhary MA. Vaccination against hepatitis $B$ among doctors at a teaching hospital in Lahore. Pak J Med Sci. 2001; 17:229-32.

8. Luiz A, Ciorlia S, Dirce MT, Zanetta. Hepatitis B in health care workers: prevalence, vaccination and relation to occupational factors. Braz J Infect Dis. 2005.

9. Mehrdad A, Leila M. The prevalence of needle stick injuries in medical, dental, nursing and midwifery students at the university teaching hospital of Shiraz, Iran. Indian J Med Sci. 2000; 60: 
227-232.

10. Imam SF, Haroon M, Amir R. Status of Immunization against hepatitis $B$ virus infection among health care workers. Biomedica. 2000;16:25-7.

11. Bhatti TA, HBV, HCV and HIV in blood donors of Liaquat University Hospital. Abstract. Pakistan Society of Gastroenterology. 2005.

12. Rehman K, Khan AA, Haider Z, Shahzad A, Iqbal $J$, Khan R, et al. Prevalence of seromarkers of $\mathrm{HBV}$ and HCV in health care personnel and apparently healthy blood donors. JPMA 1996; 46:152-4.

13. Pirzado ZA, Malik R, Rathi SL, Sheikh SA, Sheikh WM. Prevalence of hepatitis $B$ carriers in
Chandka Medical College and Hospital staff, Larkana. Pak J Med Res. 1996; 35: 183-4.

14. Khan GM, Malik MN, Rana K, Fayyaz A. Profile of hepatitis $B$ surface antigen positivity in health care personnel. Mother Child. 1996; 34: 135-8.

15. Jabeen $M$, Hussain $R$, Siddiqui $S A$, Fayyaz $A$, Saleem M. Prevalence of hepatitis B surface antigen (HBsAg) in doctors, medical students and paramedical staff working in Sir Ganga Ram Hospital, Lahore. Pak J Med Res. 1995; 35:172-5.

16. Shaikh MH, Shams K. Prevalence of HBV markers in health care personnel vs. matched controls. J Coll Physicians Surg Pak. 1995; 5: 19-21.

AUTHOR AFFILIATION:

Dr. Muhammad S Memon (Corresponding Author)

Associate Professor

Department of Medicine,

Isra University Hospital Hyderabad, Sindh - Pakistan

Email: sadik369@yahoo.com

\section{Dr. Shoaib Ansari}

Senior Lecturer

Department of Medicine

Liaquat University of Medical \& Health Sciences (LUMHS)

Jamshoro, Sindh - Pakistan.

\section{Dr. Rashid Nizamani}

House Officer

Department of Surgery,

LUMHS, Jamshoro, Sindh - Pakistan.

\section{Dr. Naresh Kumar Khatri}

Research Medical Officer

Department of Medicine

Isra University Hospital Hyderabad, Sindh - Pakistan.

\section{Prof. Mukhtar A Mirza}

Department of Medicine

Isra University Hospital Hyderabad, Sindh - Pakistan.

\section{Prof. Wasim Jafri}

Department of Medicine,

The Aga Khan University Hospital, Karachi - Pakistan. 